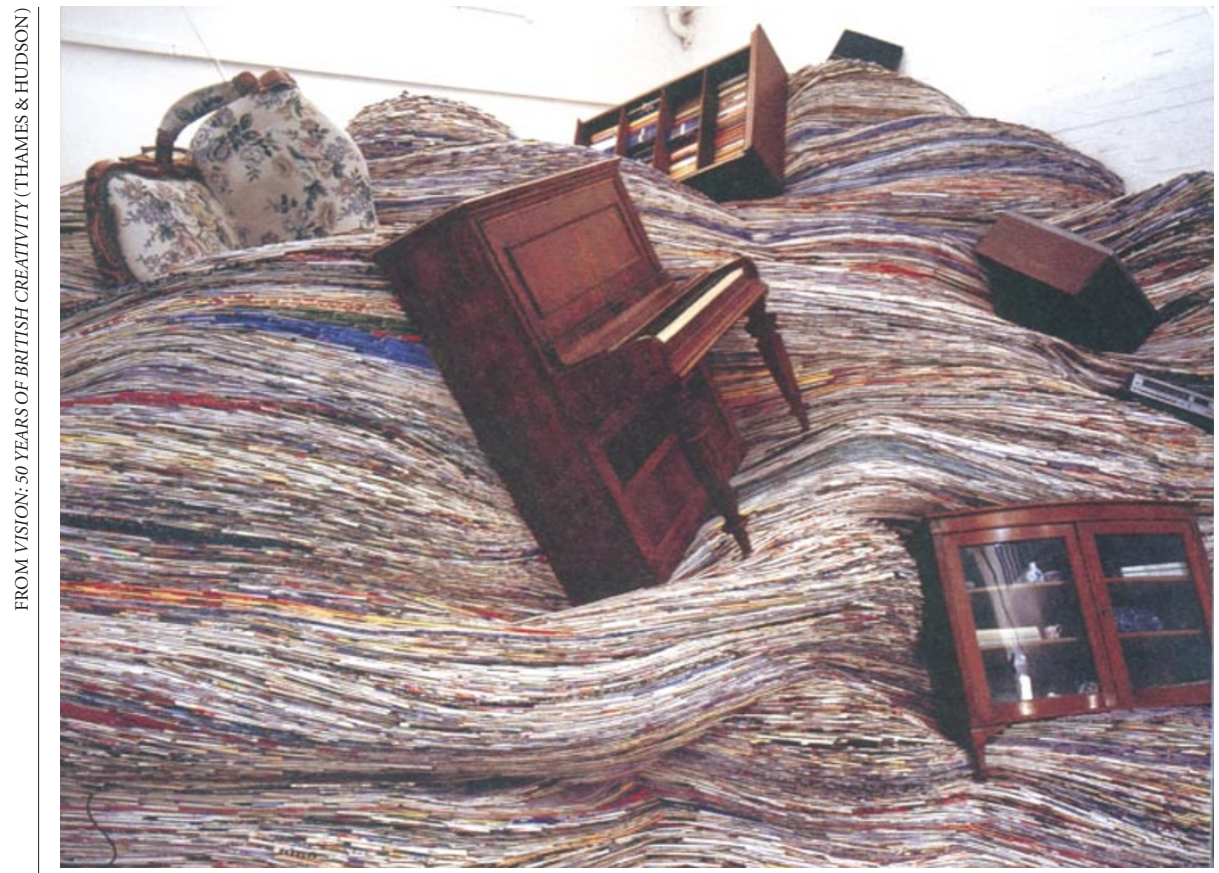

Wiring up: neural-network models have thrown light on how we perceive and experience our world.

To the extent that the models can be related to brain systems, they can be tested and, eventually, the chasm that once separated the mind and the brain can be bridged.

One of the most surprising and puzzling recent discoveries in brain science has been the degree to which the cerebral cortex, the most highly evolved part of the human brain, remains malleable. The surface of the body is mapped onto the surface of the cortex in such a way that nearby points on the body map onto nearby neurons in the cortex. When a sensory nerve that innervates the fingers of a monkey is cut, the map reorganizes and the cortical area once dedicated to that patch of the body surface is, over time, reassigned to neighbouring body surfaces. This process involves both cortical and subcortical mechanisms for neural plasticity. Conversely, when a monkey is asked to use its fingers repeatedly over a long period, the area devoted to those fingers enlarges. Something similar happens in the somatosensory cortex of human Braille readers.

Neural-network models of cortical maps with Hebbian synaptic plasticity on the sensory inputs can reproduce the changes that occur during loss of neuronal input. Shortly after limb amputation in humans, vivid phantom sensations can occur, often accompanied by intractable pain that is referred to the missing limb. Curiously, reports of phantom limbs are rare following spinal injury leading to paraplegia. Why should these two ways of cutting off neuronal input to the cortex have such different perceptual consequences?

One possibility, explored by Spitzer in a cortical model, assumes that there is noise in the stump of the severed nerve. In the model, the noise excites cortical neurons and leads to cortical reorganization but, in the case of spinal injury where it is assumed that such noise is absent or reduced, the reorganization of the map in the model is attenuated. The advantage of the model is that plausible hypotheses can be generated and explored in a system whose complexity escapes direct reasoning.

Reading this book was like visiting an old friend after a long absence. The friend is ageing gracefully but has taken up new hobbies. Since these simple neural-network models were introduced we have learned much more about the brain, and a new generation of neural-network models has been explored, based on the detailed biophysical properties of neurons. Despite this greater sophistication, many of the concepts that emerged from the first generation of neural-network models are still applicable, such as attractor states and population codes. One major difference is a richer dynamic by virtue of the intrinsic properties of the neurons. With much faster computers it has also been possible to simulate more neurons and to test how the dynamical states scale with the size of the network.

Perhaps the most puzzling property that these new network models exhibit, mimicking the brain, is spontaneously active oscillations. New concepts are needed to explain the complex mixtures of rhythms that occur in these networks and to characterize their computational power. Perhaps ten years from now in a second edition Spitzer or his students will draw out the consequences of these new models for psychology and psychiatry in an equally clear and effective way.

The last chapter contains a brief "User's manual for your brain”. Just as cardiologists can provide advice on diet and exercise to enhance your physical well-being, Spitzer provides a mental diet and exercise that may enhance brain development in children and maintain a sharp mental edge in adults. It should be possible to expand this user's manual as further discoveries are made about the brain and integrated into a clearer picture of how brains are influenced by the world.

Freud provided a language for organizing human experience that lasted nearly 100 years. A new language of the mind is now emerging from cognitive neuroscience and computer models that may have far-reaching consequences for how we see ourselves.

Terrence J. Sejnowski is at the Salk Institute and in the Department of Biology, University of California, San Diego, California 92093, USA.

\section{The first three billion years}

\section{Cradle of Life: The Discovery of Earth's Earliest Fossils}

by J. William Schopf

Princeton University Press: 1999.367 pp.

$\$ 29.95, \mathfrak{E} 17.95$

\section{Stefan Bengtson}

Of all the fossils on Earth, some have to be the earliest. So what's the big deal, except for an entry in The Guinness Book of Records? Quite a big deal, in fact. The Phanerozoic - the 'time of visible [animal] life' of the past 550 million years - is now known to have been preceded by three billion years or more of Precambrian life, almost exclusively microbial. It seems that life appeared as soon as conditions at all permitted it. Life may well be (to use Christian de Duve's words) a cosmic imperative.

Bill Schopf has been a prime mover on this frontier, in his quest to find microfossils by slicing up uncountable chunks of the Earth's older crust and in his messianic efforts to bring together scientists of all creeds and talents to ask: 'What does it all mean?' His persistent questioning, arguing, pleading, shouting, bullying, persuading, fund-raising, entertaining, writing and editing have probably promoted and inspired more interdisciplinary work on the history of life than any other factor.

In the well-written Cradle of Life, Schopf tells his story of how Earth's early microbial biosphere was discovered. He ranges from biochemistry to natural history, science history and personal anecdotes, and although the path can be tortuous, it is not too convoluted. The many diagrams, however, usually lack information about data sources; this is particularly troublesome, as it is apparent that some plots are based on idealized data.

Schopf's treatment of the earlier players in the field is balanced and entertaining, but currently active players hardly get a mention. One could argue with some of the judge- 
ments. A. C. Seward is (dis)credited with stifling research in the field for almost 40 years through his 1931 critique of the known or asserted Precambrian fossil record. If Seward's call for "more critical examination of the evidence" indeed had such an effect, surely the blame lies with those who might have taken up the challenge but didn't.

Nor does Schopf spare himself; the account of how, in 1965, he helped his professor take advantage of a peer-review commission to get the edge on a competitor would raise more than eyebrows if the act were committed today. The 1983 paper by Awramik, Schopf and Walter, reporting the world's oldest fossils from the Warrawoona Group in western Australia, is called a fiasco, because the original sampling spot could not be found; Schopf's own 1993 paper is credited with the real discovery. However, the earlier work showed that these fossils existed, and so was no more a fiasco than Christopher Columbus's voyage (about which there was also uncertainty over exact locations). In this vein, Schopf's paper rather compares to Amerigo Vespucci's voyages. Isn't that good enough?

The book is not free from bloopers, which could have been avoided by more careful reviewing. The map of North America on page 37 should be deeply offensive to those of a Canadian persuasion in New Brunswick and Nova Scotia; the Bolsheviks are credited with seizing power from the czar (who had actually abdicated more than half a year before the Bolshevik coup); and atmospheric carbon dioxide is said to act "like the windows of a greenhouse, it holds in heat, storing it in the chemical bonds that knit its atoms together" (no, it absorbs infrared radiation and converts it to heat in the atmosphere; greenhouse windows mostly just keep warm air in).

A central message of the book is that Precambrian biology differs fundamentally from Phanerozoic biology. This is indeed so; the transition between the two stands out as the greatest revolution in the history of the biosphere. Schopf makes a claim for 'hypobradytely' - extremely slow evolution during the Precambrian, owing to the predominance of asexual reproduction, "a new fundamental insight that ... stands out as one of the most striking findings ... since Darwin". Whether or not this bold claim will stand the test of time, the measurement of evolutionary rates in fossil microorganisms faces formidable difficulties, because in general only morphology is preserved, and poorly so.

Another obstacle to understanding early fossils is the bias imposed by the small subset of life-forms that have happened to survive until today. By using living organisms as our models (for example, by identifying the earliest fossils as cyanobacteria, as Schopf does), we may blind ourselves to the host of extinct organisms, and unwittingly introduce hypo- bradytely as a taxonomic artefact.

This problem follows us into astrobiology; we need methods to recognize life-forms, fossil or living, that may be totally unrelated to those on Earth. In an epilogue on that theme, Schopf mischievously places a chapter on two of the most famous howlers in palaeontology — Scheuchzer's 'Homo diluvii testis' and Beringer's 'Lügensteine' side-by-side with a critical discussion and effective debunking of the claim for ancient life in the Martian meteorite ALH84001 (Schopf was the invited sceptic at the NASA press conference in 1996). In no way does he deride the field, however. Like Seward 65 years earlier, Schopf has advocated constructive scepticism in a field of research where even the existence of the subject is in doubt. Like Seward, he deserves praise for this.

Stefan Bengtson is in the Department of Palaeozoology, Swedish Museum of Natural History, Box 50007, SE-104 05 Stockholm, Sweden.

\section{Damsels and dragons}

\section{Dragonflies: Behaviour and Ecology of Odonata}

by Philip S. Corbet

Harley: 1999.882 pp. $£ 62.50$

\section{Mike Siva-Jothy}

Dragonflies have almost certainly been using the same habitats in the same way for more than 250 million years. But, despite the general conservatism in this insect order, there is considerable variation in many aspects of their larval and adult behaviour, much of which is influenced by ecology. Philip Corbet's book is a rare beast: a truly authoritative natural history monograph dealing with a large taxon, and not strictly the behavioural ecology text that the title suggests.

Almost 20 years ago, as a student, I heard a rumour that Corbet, the patriarch of odonatologists, was about to write a new book. As the years passed, expectations grew, but later turned into concern that perhaps the work would not be finished. Key contributors to the material and impetus for the book passed away while it was in utero, including the extraordinary Peter Miller, whose research contributes something to every chapter. Not surprisingly, when the book eventually appeared, there was concern that it would not live up to 18 years of anticipation. But it did. Every aspect of biology touched upon is explained clearly and carefully by an author who has truly made the effort to understand it all.

Corbet has synthesized a phenomenal amount of material. He deliberately does not organize the book around the currently important questions in evolutionary ecology. Instead he has written a cleverly cross-referenced and indexed text that provides all the

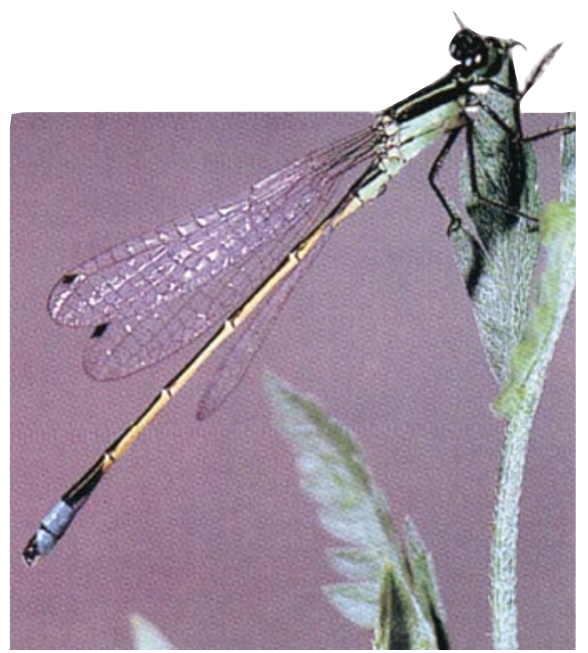

raw material from which ideas can be developed, or key information sought. The value in this approach is that, as current questions are replaced, the book will still provide wellorganized information.

The book follows the sequence of the key life-history stages, starting with eggs, moving through larval behaviour and life-history traits, then dealing with emergence, preadult development and, in the largest chapter, reproduction. This is the most complete synthesis of behavioural, ecological and background material from a single taxonomic group I have ever seen. As though it were not enough to synthesize so much information, Corbet has also provided a large set of appendices giving valuable follow-up material from each chapter.

Corbet never pulls his linguistic punches, and uses strictly correct entomological terms throughout. This is initially daunting (as is the uncompromising use of binomial nomenclature), but the reasoning behind it is important: these terms are increasingly falling into disuse as well as misuse. The glossary at the end is invaluable.

I have only two quibbles. First, most of the figures are directly reproduced from their original sources, which means sometimes there is a hotchpotch of illustrative styles that does not always do justice to the text. Second, an illustrated key of the major taxa would have been a useful foil to the abundance of binomial names. But these criticisms are pebbles, thrown at an edifice that will sit next to Bert Hölldobler and Edward O. Wilson's The Ants (Springer, 1990) as one of the definitive natural history texts of the twentieth century. Mike Siva-Jothy is in the Department of Animal and Plant Sciences, University of Sheffield, Sheffield S10 2TN, UK.

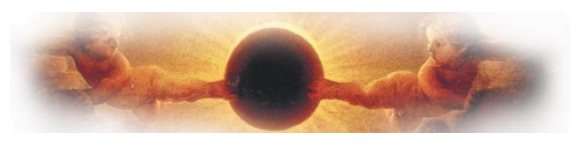

correction In the review "A user's guide to two minutes of totality" by Jay M. Pasachoff (Nature 399, 651-652; 1999), the photograph of the total eclipse of the Sun was taken by the Extreme-ultraviolet Imaging Telescope Team, Solar and Heliospheric Observatory, NASA and ESA. 